\title{
Tidal Dynamics of Relativistic Flows Near Black Holes
}

\author{
C. Chicone \\ Department of Mathematics \\ University of Missouri-Columbia \\ Columbia, Missouri 65211, USA \\ B. Mashhoon* \\ Department of Physics and Astronomy \\ University of Missouri-Columbia \\ Columbia, Missouri 65211, USA
}

\begin{abstract}
We point out novel consequences of general relativity involving tidal dynamics of ultrarelativistic relative motion. Specifically, we use the generalized Jacobi equation and its extension to study the force-free dynamics of relativistic flows near a massive rotating source. We show that along the rotation axis of the gravitational source, relativistic tidal effects strongly decelerate an initially ultrarelativistic flow with respect to the ambient medium, contrary to Newtonian expectations. Moreover, an initially ultrarelativistic flow perpendicular to the axis of rotation is strongly accelerated by the relativistic tidal forces. The astrophysical implications of these results for jets and ultrahigh energy cosmic rays are briefly mentioned.
\end{abstract}

PACS numbers: $04.20 . \mathrm{Cv}, 97.60 . \mathrm{Lf}, 98.58 . \mathrm{Fd}, 98.70 . \mathrm{Sa}$

\section{Introduction}

This paper is about the motion of free test particles in the background gravitational field of a Kerr black hole. The geodesics of Kerr spacetime are well known and have been extensively studied in standard systems of coordinates such as the Boyer-Lindquist coordinate system. The Boyer-Lindquist

\footnotetext{
${ }^{*}$ Corresponding author. E-mail: mashhoonb@missouri.edu (B. Mashhoon).
} 
coordinates are adapted to the stationary and axisymmetric nature of the source and are Minkowskian (expressed in spherical polar coordinates) infinitely far from the black hole. We are interested however in the relative motion of free nearby particles; as explained in detail in [1, from the standpoint of relativity theory the study of relative motion is certainly preferred, since it is in keeping with the spirit of the theory. Moreover, relative motion has direct observational significance. Thus theoretical results obtained in the study of relative motion can be directly compared with suitable experimental data [1, 2].

To study the relative motion of free particles, we need a reference particle whose motion is well known. For the purposes of the present work, we select the free motion of particles on radial escape trajectories along the rotation axis of the Kerr black hole. Thus at a given initial position along a radial direction in Schwarzschild spacetime or the rotation axis in Kerr spacetime, the reference particle has an escape velocity such that the particle eventually reaches infinity with zero kinetic energy. We then study the relative motion of the relativistic particles that start from the same location as the reference particle and travel in different directions. The origin of these relativistic particles on the axis of rotation will not be discussed in this paper. We will simply assume that such particles exist near the poles of the Kerr black hole. The nature of the central engine and the details of the accretion phenomena that could generate such relativistic particles are beyond the scope of this work. Instead, we concentrate on the gravitational dynamics of these particles relative to the reference particle.

To characterize the relative motion, we establish a quasi-inertial coordinate system along the worldline of the reference particle. This Fermi normal coordinate system [3] is valid in a cylindrical region of radius $\mathcal{R}$ along the worldline of the reference particle. Here $\mathcal{R}$ is the radius of curvature of the spacetime manifold. The geodesic equations of motion for the free particles are then integrated in this Fermi coordinate system subject to the limitation that the relative distance must remain well within the admissible range of Fermi coordinates. The results of such an investigation of the relative motion of free particles would naturally depend on the choice of the reference particle. For instance, a separate investigation would be necessary if the reference trajectory is an orbit in the equatorial plane of the Kerr black hole. In fact, a judicious choice of the reference particle would be essential to explain a particular observational result. Moreover the relative distance in our approach should be less than $\mathcal{R}$.

We find that ultrarelativistic flows in the gravitational field of collapsed configurations can exhibit purely non-Newtonian behavior due to general 
relativistic tidal effects. These include the phenomenon of the tidal deceleration along the axis of rotation and the phenomenon of tidal acceleration perpendicular to the axis of rotation, which follow from the integration of the generalized Jacobi equation [1, 2]. We study this equation and its extension in this paper and compare our results with the corresponding nonrelativistic tidal effects. In the following we use units such that $c=1$.

Imagine a geodesic worldline in the gravitational field of an external source. Let $u^{\mu}=d x^{\mu} / d \tau$ be the four-velocity vector of this reference geodesic and $\tau$ be its proper time. A neighboring geodesic with an arbitrary velocity relative to the reference geodesic is connected to it by the deviation vector $\xi^{\mu}(\tau)$ such that $\xi^{\mu} u_{\mu}=0$. The generalized Jacobi equation [1] is given by the geodesic deviation equation to first order in $\xi^{\mu}$, namely,

$$
\begin{aligned}
& \frac{D^{2} \xi^{\mu}}{D \tau^{2}}+R_{\rho \nu \sigma}^{\mu} u^{\rho} \xi^{\nu} u^{\sigma} \\
& +\left(u^{\mu}+\frac{D \xi^{\mu}}{D \tau}\right)\left(2 R_{\zeta \rho \nu \sigma} u^{\zeta} \frac{D \xi^{\rho}}{D \tau} \xi^{\nu} u^{\sigma}+\frac{2}{3} R_{\zeta \rho \nu \sigma} u^{\zeta} \frac{D \xi^{\rho}}{D \tau} \xi^{\nu} \frac{D \xi^{\sigma}}{D \tau}\right) \\
& +2 R_{\rho \nu \sigma}^{\mu} \frac{D \xi^{\rho}}{D \tau} \xi^{\nu} u^{\sigma}+\frac{2}{3} R_{\rho \nu \sigma}^{\mu} \frac{D \xi^{\rho}}{D \tau} \xi^{\nu} \frac{D \xi^{\sigma}}{D \tau}=0 .
\end{aligned}
$$

Here $D \xi^{\mu} / D \tau=\xi_{; \nu}^{\mu} u^{\nu}$ is the covariant derivative of $\xi^{\mu}$ along the reference worldline. Let $\lambda_{(\alpha)}^{\mu}$ be an orthonormal tetrad frame that is parallel propagated along the reference trajectory such that $\lambda_{(0)}^{\mu}=u^{\mu}$. Then, $\xi^{\mu}=X^{i} \lambda^{\mu}{ }_{(i)}$ and equation (10) may be written in terms of Fermi coordinates ( $T, \mathbf{X})$, where $T=\tau$ along the reference geodesic, as [1, 2]

$$
\begin{aligned}
& \frac{d^{2} X^{i}}{d T^{2}}+{ }^{F} R_{0 i 0 j} X^{j}+2{ }^{F} R_{i k j 0} V^{k} X^{j} \\
& +\left(2^{F} R_{0 k j 0} V^{i} V^{k}+\frac{2}{3}{ }^{F} R_{i k j \ell} V^{k} V^{\ell}+\frac{2}{3}{ }^{F} R_{0 k j \ell} V^{i} V^{k} V^{\ell}\right) X^{j}=0 .
\end{aligned}
$$

Here $V^{i}=d X^{i} / d T,|\mathbf{V}| \leq 1$ and

$$
{ }^{F} R_{\alpha \beta \gamma \delta}(T)=R_{\mu \nu \rho \sigma} \lambda^{\mu}{ }_{(\alpha)} \lambda^{\nu}{ }_{(\beta)} \lambda^{\rho}{ }_{(\gamma)} \lambda^{\sigma}{ }_{(\delta)}
$$

is the projection of the Riemann curvature tensor on the orthonormal tetrad of the reference observer along its worldline. If the relative velocity is much smaller than the speed of light, $|\mathbf{V}| \ll 1$, the velocity-dependent terms in (11) and (2) may be neglected and the generalized Jacobi equation reduces to the standard Jacobi equation that expresses the linear evolution of the deviation between two neighboring geodesics that have negligible relative motion. This relative motion generally evolves slowly on the scale of the 
radius of curvature $\mathcal{R}$, which is defined such that $\mathcal{R}^{-2}$ is the supremum of $\left|{ }^{F} R_{\alpha \beta \gamma \delta}\right|$; therefore, $|\mathbf{X}| \ll \mathcal{R}$ in (2), since higher-order terms in the deviation equation have been neglected. In the generalized Jacobi equation, the relative velocity could approach the speed of light; therefore, the relative motion could evolve rapidly thus further restricting the temporal domain of validity of equation (2).

Equation (2) can be derived in a straightforward manner using the reduced geodesic equation in Fermi coordinates. That is, starting from the geodesic equation for a free particle in arbitrary coordinates $\hat{x}^{\mu}=\left(\hat{t}, \hat{x}^{i}\right)$,

$$
\frac{d^{2} \hat{x}^{\mu}}{d s^{2}}+\hat{\Gamma}_{\alpha \beta}^{\mu} \frac{d \hat{x}^{\alpha}}{d s} \frac{d \hat{x}^{\beta}}{d s}=0
$$

where $d s^{2}=\hat{g}_{\mu \nu} d \hat{x}^{\mu} d \hat{x}^{\nu}$, one can simply derive the reduced geodesic equation [1]

$$
\frac{d^{2} \hat{x}^{i}}{d \hat{t}^{2}}-\left(\hat{\Gamma}_{\alpha \beta}^{0} \frac{d \hat{x}^{\alpha}}{d \hat{t}} \frac{d \hat{x}^{\beta}}{d \hat{t}}\right) \frac{d \hat{x}^{i}}{d \hat{t}}+\hat{\Gamma}_{\alpha \beta}^{i} \frac{d \hat{x}^{\alpha}}{d \hat{t}} \frac{d \hat{x}^{\beta}}{d \hat{t}}=0 .
$$

Specializing this equation now to the Fermi coordinates established along the reference trajectory, i.e. $\left(\hat{t}, \hat{x}^{i}\right) \mapsto\left(T, X^{i}\right)$, and using

$$
\begin{aligned}
g_{00} & =-1-{ }^{F} R_{0 i 0 j}(T) X^{i} X^{j}+\cdots, \\
g_{0 i} & =-\frac{2}{3}{ }^{F} R_{0 j i k}(T) X^{j} X^{k}+\cdots, \\
g_{i j} & =\delta_{i j}-\frac{1}{3}{ }^{F} R_{i k j \ell}(T) X^{k} X^{\ell}+\cdots,
\end{aligned}
$$

one arrives at the tidal equation for the relative motion, since in these coordinates the reference particle remains fixed at $\mathbf{X}=0$. Restricting our attention to the terms given explicitly in (6) and thus neglecting higher-order terms of the metric in the spatial Fermi coordinates, the tidal equation reduces to the generalized Jacobi equation (2). We study the main physical consequences of this equation in the field of a Kerr black hole (see also Appendix A). Higher-order tidal accelerations are discussed in Appendix B.

Tidal deceleration along the rotation axis is discussed in the next section. The non-Newtonian character of our results is emphasized in section 3 The analysis of the generalized Jacobi equation is extended to the threedimensional case in section 4. Tidal acceleration normal to the rotation axis is discussed in section [5. Finally, section [6 contains a brief discussion of our results. 


\section{Tidal deceleration}

Imagine a Kerr source of mass $M$ and angular momentum $\mathbf{J}=M a \hat{\mathbf{z}}$ at the origin of an asymptotically inertial coordinate system $(t, x, y, z)$. Here $a>0$ is the specific angular momentum of the source. The metric of Kerr spacetime can be written in Boyer-Lindquist coordinates as

$$
\begin{aligned}
d s^{2}= & -d t^{2}+\Sigma\left(\frac{1}{\Delta} d r^{2}+d \theta^{2}\right)+\left(r^{2}+a^{2}\right) \sin ^{2} \theta d \phi^{2} \\
& +2 G M \frac{r}{\Sigma}\left(d t-a \sin ^{2} \theta d \phi\right)^{2}
\end{aligned}
$$

where $\Sigma=r^{2}+a^{2} \cos ^{2} \theta$ and $\Delta=r^{2}-2 G M r+a^{2}$. Asymptotically $(r \rightarrow \infty)$, the spherical polar coordinates $(r, \theta, \phi)$ are related to the Cartesian coordinates $(x, y, z)$ in the standard manner. In this paper, we are interested in the motion of free test particles in the stationary and axisymmetric exterior Kerr spacetime. Let us first consider geodesic motion along the axis of rotation of the source, i.e. the $z$-axis. In terms of the Boyer-Lindquist temporal coordinate $t$ and radial coordinate $r$, the motion of a free test particle along the rotation axis is given by

$$
\frac{d t}{d \tau}=\gamma \frac{r^{2}+a^{2}}{r^{2}-2 G M r+a^{2}}, \quad \frac{d r}{d \tau}= \pm \sqrt{\gamma^{2}-1+\frac{2 G M r}{r^{2}+a^{2}}},
$$

where $\tau$ is the proper time along the path and $\gamma>0$ is a constant of integration. For $\gamma<1$, the test particle cannot escape the gravitational attraction of the source. When the test particle can escape to infinity, $\gamma \geq 1$ and $\gamma$ is its Lorentz factor at spatial infinity. In the limiting case of $\gamma=1$, the test particle approaches infinity with zero speed. We assume in the following that there are many such outward moving test particles with $\gamma=1$ in the neighborhood of the rotation axis of the Kerr source forming an ambient medium around the rotating source. Relative to this ambient medium, we wish to study the motion of an ultrarelativistic jet emitted outward from a region near the source along the axis of rotation.

Consider a jet clump starting from $r_{0}$ and moving rapidly along the $z$ axis according to equation (8) with $\gamma>1$. This relative motion is properly described in the first approximation using the generalized Jacobi equation (11) expressed in Boyer-Lindquist coordinates. To compare the consequences of such an equation with astronomical data regarding the motion of jets, it is appropriate to transform this equation to a quasi-inertial Fermi normal coordinate system that is established along the reference trajectory. The explicit relationship between the Boyer-Lindquist coordinate system of Kerr 
spacetime and the Fermi system is not needed for the derivation of the equation of relative motion in Fermi coordinates (2). We therefore proceed keeping in mind that the radial coordinate $r$ of the jet clump is a function of the Fermi coordinates, $r=r(T, Z)$, but that this relationship shall remain implicit throughout this work.

Along the worldline of a generic outward moving test particle with $\gamma=1$ on the rotation axis, we establish a Fermi coordinate system $(T, X, Y, Z)$ based on an orthonormal nonrotating (i.e. Fermi-Walker transported) tetrad frame. The free test particle follows a geodesic; therefore, Fermi-Walker transport reduces to parallel transport in this case. The Fermi coordinates of the reference particle are then $(\tau, 0,0,0)$, i.e. the reference particle is at the spatial origin of the Fermi coordinates. The quasi-inertial Fermi system is the one appropriate for the comparison of the theory with observation as well as Newtonian physics [3]. In fact, the speed of a jet is determined by monitoring the motion of a jet clump with respect to neighboring "fixed" features of the ambient medium [4. The motion of the jet clump relative to the reference particle in the Fermi system is given by the tidal equation [1]

$$
\frac{d^{2} Z}{d T^{2}}+k\left(1-2 V^{2}\right) Z+O\left(Z^{2}\right)=0
$$

where $V=d Z / d T$ and the curvature $k={ }^{F} R_{0303}$ is given by

$$
k=-2 G M \frac{r\left(r^{2}-3 a^{2}\right)}{\left(r^{2}+a^{2}\right)^{3}} .
$$

Here $r$ is the Boyer-Lindquist radial coordinate of the reference particle and $r(T)$ can be determined from the integration of the second equation in (8) with $\gamma=1, \tau=T$ and $d r / d T>0$. We will be concerned with regions along the rotation axis such that $r^{2} \gg 3 M^{2} \geq 3 a^{2}$, so that $k<0$. Solving equation (8) with $\gamma=1$ and $\tau \rightarrow T$ for $r(T)$ and substituting this in equation (10) gives us the appropriate $k(T)$ to use in the tidal equation (9). Let us note that equations (8)-(10) are valid for any free reference particle moving along the rotation axis of the Kerr source.

The specific angular momentum of the source $J / M=a$ appears in equations (8) and (10) as $a^{2}$, so that these equations are invariant under the transformation $a \mapsto-a$. This implies that the sense of rotation of the black hole is of no consequence for the phenomena described in this section. This is consistent with the double-jet structure observed in astrophysics. It is therefore sufficient to treat only the motion of particles moving outward above the north pole of the black hole. 
It is important to mention here that the main results of this work are independent of the precise nature of the massive source. For the sake of concreteness, we have assumed that the source is a Kerr black hole with $a \leq G M$. Moreover, we will concentrate on a jet clump launched from $r_{0} \gg G M$ and moving along the positive $z$-axis. However, equations (8)(10) depend upon $a$ in terms of $a^{2} / r^{2}$, which is very small for $r \geq r_{0}>>a$. In fact, our main results are insensitive to the ratio $a /(G M)$. That is, our main conclusions remain the same even if the source is spherically symmetric $(a=0)$ and the jet clump is launched along a radial direction.

The tidal equation (9) in principle contains an infinite series in powers of the quantity $Z / \mathcal{R}$, where $\mathcal{R}$ is the effective radius of curvature such that $\mathcal{R}^{-2}=|k|$. For regions of interest in this work $k \approx-2 G M / r^{3}$ and hence

$$
\mathcal{R} \approx \sqrt{\frac{r^{3}}{2 G M}}
$$

Starting from $r=r_{0}$ corresponding to $Z=0$ at $T=0$, we integrate equation (9) with a given initial $V_{0}=V(0)>0$. For a highly relativistic jet clump, the initial Lorentz factor $\Gamma_{0}=\left(1-V_{0}^{2}\right)^{-\frac{1}{2}}$ is such that $\Gamma_{0} \gg 1$. The integration time $T$ must be limited such that $Z(T) \approx T<\mathcal{R}_{0}$, where $\mathcal{R}_{0}$ is given by equation (11) at $r=r_{0}$. It is important to note that the endpoint of the integration is only known implicitly in terms of the Boyer-Lindquist radial coordinate $r$. There is, of course, a definite relationship between the BoyerLindquist coordinates and the corresponding Fermi coordinates. However, this relationship remains implicit here; making it explicit would immediately imply that we know the tidal equation (9) to all orders [1].

The first-order approximation of the tidal equation corresponds to the generalized Jacobi equation,

$$
\frac{d^{2} Z}{d T^{2}}+k\left(1-2 V^{2}\right) Z=0
$$

This equation has solutions corresponding to $V= \pm 1 / \sqrt{2}$, or $\Gamma:=(1-$ $\left.V^{2}\right)^{-\frac{1}{2}}=\sqrt{2}$, where the jet moves with uniform speed relative to the reference particle. For our reference particle with $\gamma=1$, these special solutions are attractors 1, 2]. Starting near the Kerr black hole and moving along the positive $z$-axis, an initially ultrarelativistic jet clump decelerates rapidly relative to the reference particle $(\gamma=1)$ according to equation (12). This initial deceleration and approach to $1 / \sqrt{2}$ is depicted within the domain of validity of equation (12) in figures 1] and 2 for three clumps with initial Lorentz factors of $\Gamma_{0}=1000,100$ and 10 . 


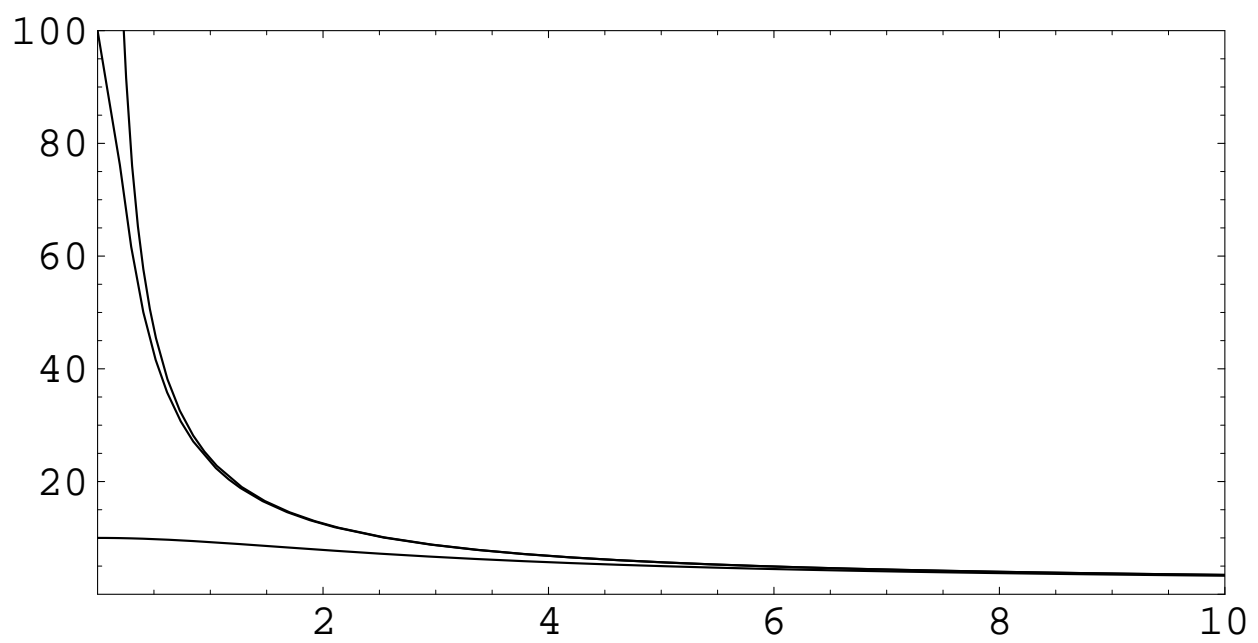

Figure 1: Plot of the Lorentz factor $\Gamma$ versus $T /(G M)$ based on the integration of equation (12) with initial data $r_{0} /(G M)=10, Z(0)=0$ and $V(0)=\sqrt{\Gamma_{0}^{2}-1} / \Gamma_{0}$ for $a /(G M)=1$. The graph illustrates the deceleration of clumps with initial Lorentz factors $\Gamma_{0}=1000,100$ and 10.

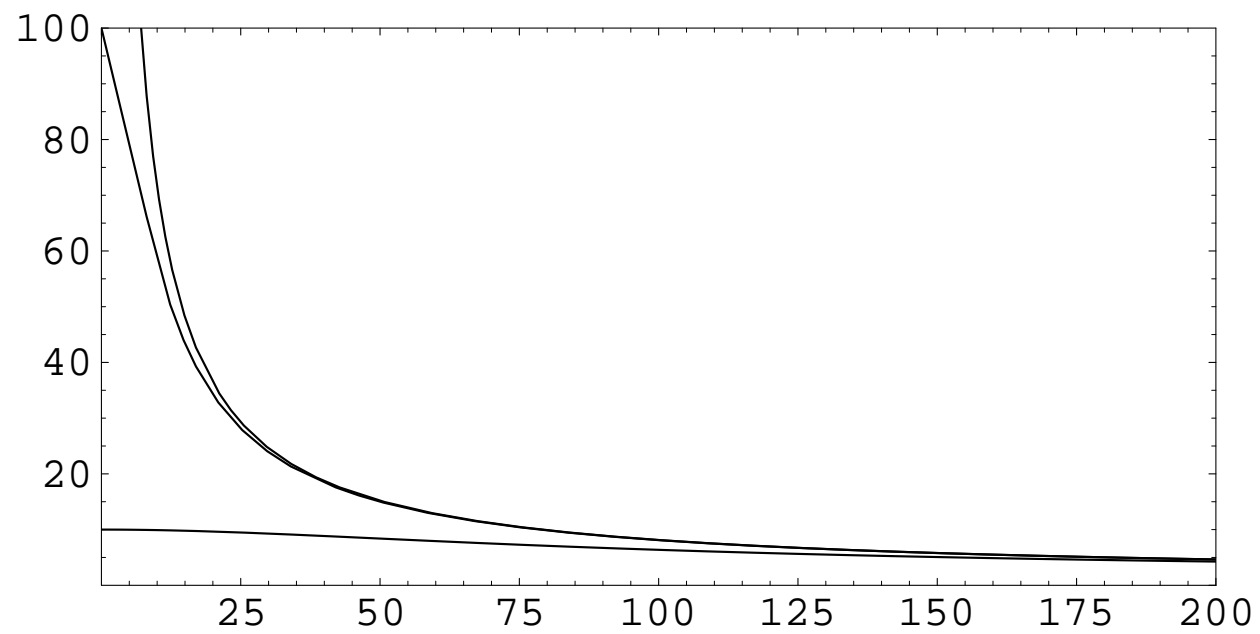

Figure 2: Plot of the Lorentz factor $\Gamma$ versus $T /(G M)$ based on the integration of equation (12) with initial data $r_{0} /(G M)=100, Z(0)=0$ and $V(0)=\sqrt{\Gamma_{0}^{2}-1} / \Gamma_{0}$ for $a /(G M)=0$. The graph illustrates the deceleration of clumps with initial Lorentz factors $\Gamma_{0}=1000,100$ and 10. 
The critical speeds $\pm 1 / \sqrt{2}$ that appear in equation (12) correspond to the critical Lorentz factor $\Gamma_{c}=\sqrt{2}$. A particle of mass $m$ with a Lorentz factor of $\Gamma_{c}$ has kinetic momentum $m$, hence its de Broglie wavelength equals its Compton wavelength. For our present purposes, the motion of a particle is ultrarelativistic if its corresponding Lorentz factor $\Gamma$ exceeds $\Gamma_{c}$. For $\Gamma \ll \Gamma_{c}$, the tidal equation has already been extensively studied in the field of a Kerr black hole [5, 6].

The novel deceleration effect is thus a consequence of equation (21) restricted to ultrarelativistic motion along a certain straight line. It is therefore interesting to consider the application of the generalized Jacobi equation to the motion of astrophysical jets [1, 2. Thus imagine a rotating gravitational source with a double jet system moving outward along its axis of rotation. We have assumed that the Fermi coordinates established along an ambient trajectory that lies on the axis of rotational symmetry of the source are such that the axis of rotation is the $Z$-axis. Concentrating on an ultrarelativistic jet moving along the positive $Z$-axis, one finds that equation (2) in this case involves a remarkably strong initial deceleration followed by a gradual decline toward a terminal speed given by $1 / \sqrt{2}$. Starting with an ultrarelativistic jet, this terminal speed is approached asymptotically after an infinite period of time. On the other hand, the generalized Jacobi equation is only valid for a rather limited timescale that nevertheless covers the initial rapid drop in velocity.

Figures 1 and 2 illustrate the main result of this paper regarding tidal deceleration; therefore, it is worthwhile to develop an approximate analytic understanding of how this tidal deceleration comes about. To fix our ideas, we focus attention in what follows on a jet clump with an initial Lorentz factor $\Gamma_{0} \gg 1$ moving along the positive $z$-direction relative to a free test particle of the ambient medium. At $T=0, Z=0$, i.e. the jet starts from the position of the reference particle on the $z$-axis, where

$$
r \approx\left(r_{0}^{3 / 2}+\frac{3}{2} \sqrt{2 G M} T\right)^{2 / 3}
$$

according to equation (8) with $\gamma=1$ and $r(T=0)=r_{0} \gg 2 G M$. It follows that in equation (91),

$$
k \approx-2 \frac{G M}{\left(r_{0}^{3 / 2}+\frac{3}{2} \sqrt{2 G M} T\right)^{2}} .
$$

Starting from equation (12) and writing $d^{2} Z / d T^{2}=V d V / d Z$, it is simple to show that equation (12) can be completely integrated for a constant 
curvature $k$; in fact, the phase diagram for this case has been given schematically in figure 2 of [1]. For $k=k(T)$, however, let us note that

$$
\frac{d}{d T} \ln \left(\frac{1-2 V^{2}}{1-2 V_{0}^{2}}\right)=4 k(T) V Z
$$

Since $k<0$, it follows form equation (15) that if $V_{0}^{2}<1 / 2, V^{2}$ increases toward $1 / 2$, while if $V_{0}^{2}>1 / 2, V^{2}$ decreases toward $1 / 2$. Integrating equation (15), we find

$$
\ln \left(\frac{1-2 V^{2}}{1-2 V_{0}^{2}}\right)=4 \int_{0}^{T} k\left(T^{\prime}\right) V\left(T^{\prime}\right) Z\left(T^{\prime}\right) d T^{\prime}
$$

To find an approximate solution of this equation we write equation (16) in the form

$$
\ln \left(\frac{1-2 \Gamma^{-2}}{1-2 \Gamma_{0}^{-2}}\right)=4 \int_{0}^{T} k\left(T^{\prime}\right) V\left(T^{\prime}\right) Z\left(T^{\prime}\right) d T^{\prime} .
$$

Assuming that $\Gamma \gg 1$ and $\Gamma_{0} \gg 1$, the left side of equation (17) can be approximately written as $2\left(\Gamma_{0}^{-2}-\Gamma^{-2}\right)$. Next, in the integrand of equation (17) we let $V\left(T^{\prime}\right) \approx 1$ and $Z\left(T^{\prime}\right) \approx T^{\prime}$, and note that

$$
\int_{0}^{T} k\left(T^{\prime}\right) T^{\prime} d T^{\prime} \approx-\frac{4}{9}\left[\ln (1+\lambda)+\frac{1}{1+\lambda}-1\right]
$$

using equation (14). Here $\lambda$ is defined by

$$
\lambda=\frac{3}{2} \frac{\sqrt{2 G M} T}{r_{0}^{3 / 2}},
$$

so that $2 \lambda / 3=T / \mathcal{R}_{0}$, using equation (11). For $\lambda \ll 1$, one can show that

$$
\ln (1+\lambda)+\frac{1}{1+\lambda} \approx 1+\frac{1}{2} \lambda^{2}+O\left(\lambda^{3}\right) .
$$

Equations (17)-(20) thus imply that

$$
\Gamma^{-2} \approx \Gamma_{0}^{-2}+\left(\frac{T}{\mathcal{R}_{0}}\right)^{2}
$$

or $V^{2} \approx V_{0}^{2}-\left(T / \mathcal{R}_{0}\right)^{2}$. This result provides an approximate solution of equation (12) for $\Gamma_{0} \gg 1, \Gamma \gg 1$ and $T \ll 2 \mathcal{R}_{0} / 3$. Indeed, a graph of equation (21) is almost indistinguishable from the corresponding numerical solution of equation (12) presented in figures 1 and 2 . 
To get an estimate of the time $T$ that it would take for a highly relativistic jet clump with $\Gamma_{0} \gg 1$ to decelerate to $\Gamma$, where $\Gamma_{0}^{2} \gg \Gamma^{2} \gg 1$, we note that $\lambda \approx 3 /(2 \Gamma)$ once we neglect $\Gamma_{0}^{-2}$ compared with $\Gamma^{-2}$. Thus equation (21) can be written as

$$
\frac{T}{G M} \approx \frac{1}{\Gamma} \sqrt{\frac{1}{2}\left(\frac{r_{0}}{G M}\right)^{3}} .
$$

It follows that the deceleration time is essentially independent of $\Gamma_{0}$. This remarkable result is already evident from the form of equation (17): for $\Gamma_{0} \gg \Gamma \gg 1$, the left side of equation (17) is nearly independent of $\Gamma_{0}$. In figure 1 for instance, $r_{0}=10 G M$ and the time it takes for $\Gamma$ to decrease to $\Gamma \approx 5$ from any initial $\Gamma_{0} \gg 5$ should be $\approx 2 \sqrt{5} G M$ based on equation (22), in good agreement with the numerical results of figure 1. Thus no matter how relativistic the clump may be initially at $r_{0}=10 G M$, it decelerates to $\Gamma \approx 5$ in a short time of order $G M$. Moreover, this time is within the limit of validity of the generalized Jacobi equation, since equation (22) may be written as

$$
\frac{T}{\mathcal{R}_{0}} \approx \frac{1}{\Gamma} \ll 1 .
$$

The difference between figure1 and figure 2 is a reflection of the fact that the curvature $k$ decreases as the inverse cube of the radial distance $(r \gg G M)$ from the black hole and, using equation (22) for a fixed $\Gamma, T^{2} \propto r_{0}^{3}$, which is reminiscent of Kepler's third law.

Thus far we have considered only one reference particle of the ambient medium with $\gamma=1$. Let us now imagine $N$ such particles all with $\gamma=1$ distributed along the rotation axis of the source and constituting "fixed" features of the ambient medium that can be used for reference purposes. More specifically, let the Boyer-Lindquist radial coordinates $r_{i}, i=1,2, \ldots, N$, of the particles be such that $r_{1}<r_{2}<\ldots<r_{i}<r_{i+1}<\ldots<r_{N}$; moreover, each interval is small enough that the generalized Jacobi equation is valid within this interval to a reasonably good approximation. That is, $N$ different Fermi frames can be established that are centered on the $N$ particles and the generalized Jacobi equation can be applied separately within each interval $\left[r_{i}, r_{i+1}\right]$, so that if the initial speed of the jet clump relative to $r_{i}$ is more than the terminal speed, the clump continues to decelerate within this interval, and so on. This is a direct consequence of the remarkable feature of equations (8)-(10) noted above: they do not explicitly depend on any specific reference particle with $\gamma=1$. In this sense, the clump decelerates with respect to the ambient medium toward the terminal speed. As the jet clump approaches the terminal speed, the first-order tidal term proportional to $1-2 V^{2}$ in equation (9) approaches zero and hence the higher-order tidal 
terms, neglected in equation (9), become dominant. A complete knowledge of these terms is then required to predict the subsequent evolution of the flow. It may be that at such a time the clump is sufficiently far from the black hole that tidal effects become unimportant and plasma forces take over the dynamics of the clump [2].

Consider now the tidal equation (9) in the nonrelativistic limit $|V| \ll$ 1. The first-order approximation leads in this case to the standard Jacobi equation, i.e. equation (12) but with $1-2 V^{2}$ replaced by unity. As explained before, for a clump along the rotation axis at $r$ with $r^{2} \gg 3 a^{2}, k \approx-2 G M / r^{3}$ just as in Newtonian mechanics. The tidal force of the central source in this case simply tends to pull the clump and the reference particle apart, leading to a relative tidal acceleration. The result of the integration of the standard Jacobi equation is in this case essentially the same as in the Newtonian theory [5, 6], which is studied in the next section.

\section{Comparison with Newtonian gravitation}

It is useful to compare the main results of the relativistic approach with the Newtonian theory of tides, since our main conclusions regarding tidal deceleration and terminal speed have no counterparts in the Newtonian approach and hence violate our "nonrelativistic" intuition.

Let us consider in the background inertial coordinate system $(t, x, y, z)$, the Newtonian equations of motion of the jet clump at $r$ and the reference particle at $r_{p}$ along the rotation axis of the source

$$
\frac{d^{2} r}{d t^{2}}=-\frac{G M}{r^{2}}, \quad \frac{d^{2} r_{p}}{d t^{2}}=-\frac{G M}{r_{p}^{2}}
$$

We are interested in the relative motion; therefore, with $\zeta=r-r_{p}$,

$$
\frac{d^{2} \zeta}{d t^{2}}=-\frac{G M}{\left(r_{p}+\zeta\right)^{2}}+\frac{G M}{r_{p}^{2}}
$$

For $\zeta<r_{p}$, this equation can be written as

$$
\frac{d^{2} \zeta}{d t^{2}}=\frac{2 G M}{r_{p}^{3}} \zeta+O\left(\zeta^{2}\right),
$$

which should be compared and contrasted with equation (9). The higherorder Newtonian tidal terms are considered in Appendix B 
Let us assume as before that the reference particle has zero energy, so that its kinetic and potential energies add up to zero; then, $\left(d r_{p} / d t\right)^{2}=$ $2 G M / r_{p}$ can be simply integrated and the result is

$$
r_{p}=\left(r_{0}^{3 / 2}+\frac{3}{2} \sqrt{2 G M} t\right)^{2 / 3}
$$

where $r_{p}=r_{0}$ at $t=0$. Substituting this result in equation (25), we find to linear order in $\zeta$

$$
\frac{d^{2} \zeta}{d t^{2}}-\frac{2 G M}{\left(r_{0}^{3 / 2}+\frac{3}{2} \sqrt{2 G M} t\right)^{2}} \zeta=0,
$$

which can be solved with boundary conditions that $\zeta=0$ and $d \zeta / d t=v_{0}$ at $t=0$. Here $v_{0}>0$ is the initial speed of the jet clump relative to the reference particle. The radial Newtonian tidal force tends to pull the particles apart; therefore, $\dot{\zeta}=d \zeta / d t$ monotonically increases for the duration of the validity of the approximation used here. This is the complete opposite of what happens in the relativistic theory for an initially ultrarelativistic relative speed.

An interesting feature of equation (12) is therefore that the nature of the tidal force changes depending on whether $V^{2}$ is below or above the critical value $V_{c}^{2}=1 / 2$. In fact, for $V^{2}<1 / 2$ the tides behave essentially as in Newtonian mechanics, i.e. the particle accelerates - its speed approaching $1 / \sqrt{2}$ very slowly-relative to the reference particle. The character of the tides in a model star falling into a Kerr black hole has been investigated in detail in [5, 6] using the Jacobi equation; the results are similar as in Newtonian gravitation except possibly when the model star is very close to the horizon of the black hole. On the other hand, for $V^{2}>1 / 2$ the tides behave in the opposite way (see figures 1 and 2).

Comparing the Newtonian behavior with the consequences of equations (12)-(14), we recognize the significance of the relativistic tidal acceleration term $2 k V^{2} Z$ for the difference between the results of the two theories. Thus tidal deceleration and terminal speed exhibited by (12) are due to this gravitomagnetic (i.e. motional) tidal effect that is purely relativistic and thus goes beyond Newtonian gravitation. The higher-order tidal terms [5, 6, 7] in equation (9) are treated using a certain post-Newtonian approach in Appendix B 


\section{Tidal dynamics}

It is interesting to explore the dynamics of the three-dimensional generalized Jacobi equation (2) using the same reference trajectory (8) as before. The components of the curvature tensor in the Fermi coordinate system established along this worldline are given by 8

$$
\begin{aligned}
& { }^{F} R_{0101}={ }^{F} R_{0202}=-\frac{1}{2} k, \quad{ }^{F} R_{0303}=k, \\
& { }^{F} R_{2323}={ }^{F} R_{3131}=\frac{1}{2} k, \quad{ }^{F} R_{1212}=-k, \\
& { }^{F} R_{0123}={ }^{F} R_{0231}=-\frac{1}{2} q, \quad{ }^{F} R_{0312}=q ;
\end{aligned}
$$

these are the only nonzero components except, of course, for the symmetries of the Riemann tensor. Here $(0,1,2,3)=(T, X, Y, Z), k$ is given by equation (10) and $q$ is directly proportional to the angular momentum of the source and is given by

$$
q=2 G M a \frac{3 r^{2}-a^{2}}{\left(r^{2}+a^{2}\right)^{3}}
$$

One can interpret $q$ as the "magnetic" curvature, while $k$ has the interpretation of "electric" curvature. It is a significant feature of equations (29)-(31) that they are independent of the Lorentz factor of the reference trajectory $\gamma$. This is a result of the fact that the axis of rotational symmetry in the Kerr metric provides two special tidal directions - corresponding to ingoing and outgoing trajectories - such that $k$ and $q$ are independent of any Lorentz boosts along these directions [9, 10, 11]. These special tidal directions are related to the repeated principal null directions of the curvature tensor and therefore the degenerate nature of Kerr spacetime. That is, the special tidal directions exist at each event in Kerr spacetime, since it is a vacuum spacetime of type $D$ in the Petrov classification 9, 10, 11. The significance of such directions in connection with the direction of an astrophysical jet has been pointed out in [10]: to preserve the collimation of a highly relativistic jet against tidal disruptions caused by the central source, the most natural jet direction would be a special tidal direction. Moreover, of all the special tidal directions the Kerr rotation axis has the additional advantage of being in conformity with the symmetry of the configuration under consideration. 
Using equations (29)-(31), it is possible to express equation (2) as

$$
\begin{aligned}
\ddot{X}- & \frac{1}{2} k X\left(1-2 \dot{X}^{2}+\frac{4}{3} \dot{Y}^{2}-\frac{2}{3} \dot{Z}^{2}\right)+\frac{1}{3} k \dot{X}(5 Y \dot{Y}-7 Z \dot{Z}) \\
& +q\left[\dot{X} \dot{Y} \dot{Z} X-\dot{Z} Y\left(1+\dot{X}^{2}\right)-2 \dot{Y} Z\right]=0 \\
\ddot{Y}- & \frac{1}{2} k Y\left(1-2 \dot{Y}^{2}+\frac{4}{3} \dot{X}^{2}-\frac{2}{3} \dot{Z}^{2}\right)+\frac{1}{3} k \dot{Y}(5 X \dot{X}-7 Z \dot{Z}) \\
& -q\left[\dot{X} \dot{Y} \dot{Z} Y-\dot{Z} X\left(1+\dot{Y}^{2}\right)-2 \dot{X} Z\right]=0 \\
\ddot{Z}+ & k Z\left[1-2 \dot{Z}^{2}+\frac{1}{3}\left(\dot{X}^{2}+\dot{Y}^{2}\right)\right]+\frac{2}{3} k \dot{Z}(X \dot{X}+Y \dot{Y}) \\
& -q(X \dot{Y}-\dot{X} Y)\left(1-\dot{Z}^{2}\right)=0
\end{aligned}
$$

where $\dot{X}=d X / d T$, etc. These equations reduce to the one-dimensional generalized Jacobi equation (12) for $X=Y=0$. It is simple to check that equations (33)-(35) are invariant under the transformations $(X, Y, Z ; k, q) \mapsto$ $(Y, X, Z ; k,-q)$ and $(X, Y, Z ; k, q) \mapsto(X, Y,-Z ; k,-q)$, since $q$ is directly related to the rotation of the source. Furthermore, equations (33)-(35) are also invariant under parity or time reversal if in each case we also change $q$ to $-q$.

It is interesting to note an important symmetry of the system (33)(35) under rotations about the $Z$-axis. The rotational symmetry about the direction of the motion of the reference particle implies that there is a degeneracy in the choice of the $X$ and $Y$ axes of the tetrad frame of the reference observer. Once an orthonormal tetrad is chosen, however, it is then parallel transported along the reference worldline. Nevertheless, the axial symmetry of the configuration under consideration implies that under a rotation about the $Z$-axis with a constant azimuthal angle $\phi$,

$$
\begin{aligned}
X^{\prime} & =X \cos \phi+Y \sin \phi, \\
Y^{\prime} & =-X \sin \phi+Y \cos \phi
\end{aligned}
$$

and $Z^{\prime}=Z$, the system (33)-(35) remains invariant. This symmetry can, of course, be checked directly; for instance, for $\phi=\pi / 2$ we have $X^{\prime}=Y$ and $Y^{\prime}=-X$ and it is simple to see that the transformation $(X, Y) \mapsto(Y,-X)$ leaves the system (33)-(35) invariant.

To express $k$ and $q$ as functions of the time $T$, we need an explicit solution for the trajectory (8). To this end, we set $\gamma=1$ as before and integrate $d r / d T=\sqrt{2 G M r} / \sqrt{r^{2}+a^{2}}$ with the initial condition that $r=r_{0}$ at $T=0$, where $r_{0}^{2}>3 a^{2}$. We have thus far focused our attention on an ultrarelativistic jet clump that is launched from $(X, Y, Z)=0$ at $T=0$ 
exactly along the rotation axis of the source such that $X$ and $Y$ remain zero throughout the flow. What happens if the jet is not launched exactly along the $Z$-axis? To answer this question, we must study the linearization of equations (33)-(35) about a solution $Z=Z(T)$ of equation (12).

To obtain the linearization of system (33) - [35) around the motion of an ultrarelativistic clump along the axis of symmetry, we keep only terms linear in $X, \dot{X}, Y$ and $\dot{Y}$ and find that

$$
\begin{aligned}
\ddot{X}-\frac{7}{3} k(Z \dot{Z}) \dot{X}-\frac{1}{2} k\left(1-\frac{2}{3} \dot{Z}^{2}\right) X & =q(\dot{Z} Y+2 Z \dot{Y}), \\
\ddot{Y}-\frac{7}{3} k(Z \dot{Z}) \dot{Y}-\frac{1}{2} k\left(1-\frac{2}{3} \dot{Z}^{2}\right) Y & =q(\dot{Z} X+2 Z \dot{X}),
\end{aligned}
$$

while the third equation simply reduces to equation (12).

At any given time $T$, the new system (38)-(39) represents two damped harmonic oscillators that are coupled through the magnetic curvature $q$, i.e. the coupling is due to the rotation of the source; moreover, the system decouples if it is transformed to the new variables $X \pm Y$. It is possible to obtain a simple analytic solution of this system that is linearized about the solution (22) of equation (12), which amounts to $Z(T) \approx \mathcal{R}_{0} w\left(1-w^{2} / 6\right)$ with $w=T / \mathcal{R}_{0}$. Writing

$$
k \approx-\frac{1}{\mathcal{R}_{0}^{2}}\left[1-3 w+O\left(w^{2}\right)\right], \quad q \approx 3 \frac{a}{r_{0} \mathcal{R}_{0}^{2}}\left[1-4 w+O\left(w^{2}\right)\right]
$$

for $0<w<1$, we find from equations (38) - (39) that

$$
\begin{aligned}
X(T) & =\dot{X}(0) T F\left(\frac{T}{\mathcal{R}_{0}}\right)+\frac{3}{2} \frac{a}{r_{0}} \dot{Y}(0) T H\left(\frac{T}{\mathcal{R}_{0}}\right), \\
Y(T) & =\dot{Y}(0) T F\left(\frac{T}{\mathcal{R}_{0}}\right)+\frac{3}{2} \frac{a}{r_{0}} \dot{X}(0) T H\left(\frac{T}{\mathcal{R}_{0}}\right),
\end{aligned}
$$

where only terms linear in $a / r_{0} \ll 1$ are taken into account and $F$ and $H$ are given by

$$
F(w)=1-\frac{5}{12} w^{2}+\frac{5}{8} w^{3}+O\left(w^{4}\right), \quad H(w)=w^{2}-2 w^{3}+O\left(w^{4}\right) .
$$

These results, which are approximately valid for $\Gamma_{0} \gg 1$ and $T \ll \mathcal{R}_{0}$, provide insight into the general behavior of the flow near the jet that moves along the $Z$-axis.

The axial symmetry of the system (33)-(35) under rotation about the $Z$-axis implies that it would be advantageous to express this system in cylindrical coordinates. This is done in Appendix A where an approximate constant of the motion for the system (33)-(35) is also derived based on axial symmetry. 


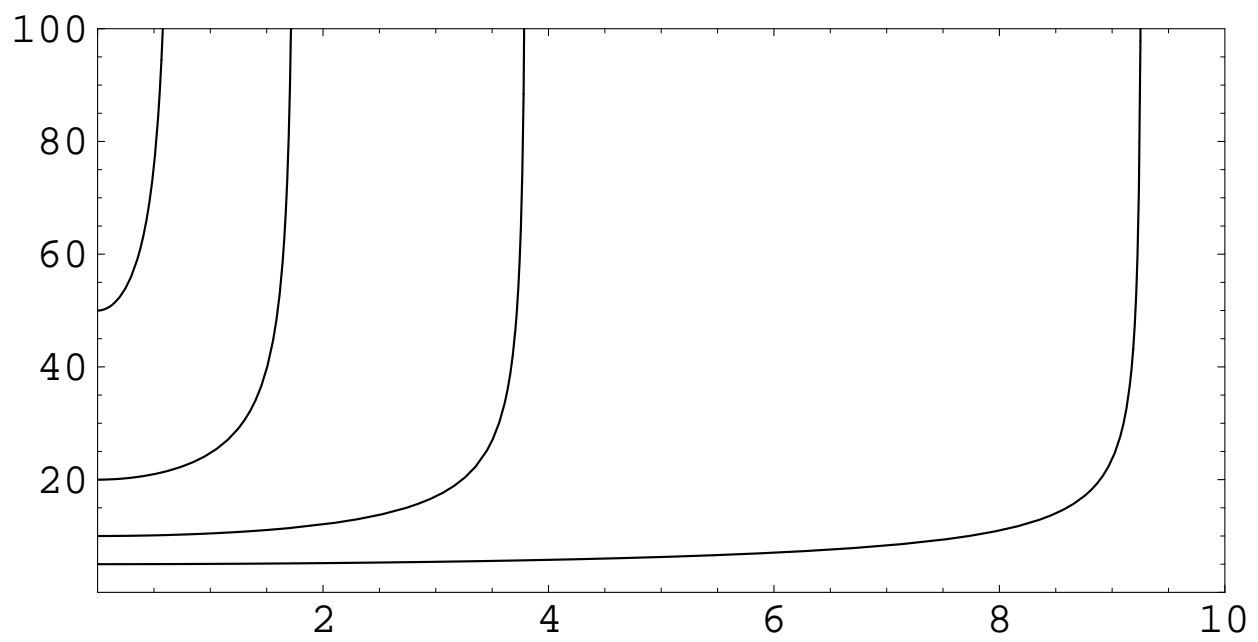

Figure 3: Plot of the Lorentz factor $\Gamma$ versus $T /(G M)$ based on integration of equation (44) with initial data $X=0$ at $T=0$ with $\dot{X}(0)=2 \sqrt{6} / 5$, $3 \sqrt{11} / 10, \sqrt{399} / 20$ and $7 \sqrt{51} / 50$ corresponding respectively to $\Gamma_{0}=5,10$, 20 and 50. In this plot $a /(G M)=1$ and $r_{0} /(G M)=10$. The graph illustrates acceleration of the particle such that $\Gamma$ essentially approaches infinity at $T /(G M) \approx 9.3,3.9,1.8$ and 0.7 for $\Gamma_{0}=5,10,20$ and 50, respectively. 


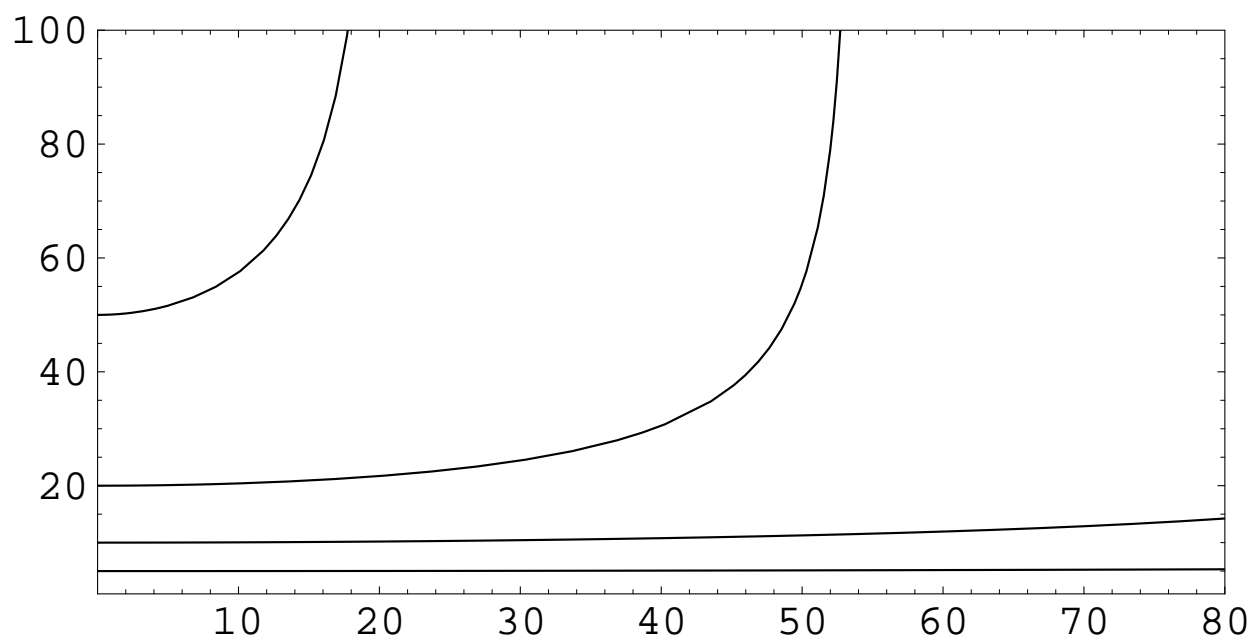

Figure 4: Plot of the Lorentz factor $\Gamma$ versus $T /(G M)$ based on integration of equation (44) with initial data $X=0$ at $T=0$ with $\dot{X}(0)=2 \sqrt{6} / 5$, $3 \sqrt{11} / 10, \sqrt{399} / 20$ and $7 \sqrt{51} / 50$ corresponding respectively to $\Gamma_{0}=5,10$, 20 and 50. In this plot $a /(G M)=0$ and $r_{0} /(G M)=100$. The graph illustrates acceleration of the particle such that $\Gamma$ essentially approaches infinity at $T /(G M) \approx 55$ and 21 for $\Gamma_{0}=20$ and 50 , respectively. 
An important feature of equations (33) - 355 is that for $Y=Z=0$, this system reduces to

$$
\ddot{X}-\frac{1}{2} k X\left(1-2 \dot{X}^{2}\right)=0 .
$$

This equation has the same form as equation (12) except that the negative curvature $k$ in (12) is replaced by $-k / 2$. It follows that a particle launched from $(X, Y, Z)=0$ along the $X$-direction with a speed above the critical speed $1 / \sqrt{2}$ is accelerated as in figures 3 and 4 . It is a consequence of the axial symmetry of the system (33)-(35) that the form of equation (44) is generic for motion along any direction perpendicular to the $Z$-axis (see Appendix A).

Equation (44) again contains the critical speed $1 / \sqrt{2}$. In this approximation, if the initial speed is below this critical value, then the particle starting with a positive initial velocity decelerates over time intervals that are much longer than those corresponding to the validity of the approximation. While the asymptotic behavior of the system has no physical meaning, one can show that the trajectories in the phase plane spiral around the origin.

If the initial speed is above the critical value, then the particle accelerates and its Lorentz factor approaches infinity in finite time. In the latter case, it is interesting to extend our approximation scheme involving equations (12)(23) in section 2 for $\Gamma_{0} \gg 1, \Gamma \gg 1$ and $T \ll 2 \mathcal{R}_{0} / 3$ to equation (44) by the simple substitution $k \mapsto-k / 2$. Instead of (21), we get

$$
\Gamma^{-2} \approx \Gamma_{0}^{-2}-\frac{1}{2}\left(\frac{T}{\mathcal{R}_{0}}\right)^{2}
$$

which implies that $\Gamma$ diverges at

$$
\frac{T_{d}}{G M} \approx \frac{1}{\Gamma_{0}}\left(\frac{r_{0}}{G M}\right)^{3 / 2}
$$

As expected, $T_{d}$ is inversely proportional to $\Gamma_{0}$ for a fixed $r_{0}$. For $\Gamma_{0}=$ 10,20 and 50 in figure 3 , equation (46) predicts that $T_{d} /(G M) \approx 3.16$, 1.58 and 0.63 , which compare favorably with $3.9,1.8$ and 0.7 obtained by the numerical integration used to produce the figure. Similar results are obtained for figure 4 for instance, $T_{d} /(G M) \approx 50$ and 20 for $\Gamma_{0}=20$ and 50, respectively, which should be compared with 55 and 21 based on numerical results presented in figure 4. The approximation (46) improves as $\Gamma_{0}$ increases. 
The phase diagram for equation (44) in the case of constant curvature is given in figure 3 of $[1$. It follows from this equation that

$$
\frac{d}{d T} \ln \left(\frac{1-2 V^{2}}{1-2 V_{0}^{2}}\right)=-2 k(T) V Z,
$$

which can be obtained from (16) by $k \mapsto-k / 2$. The left side of equation (47) is positive; therefore, if $V_{0}^{2}<1 / 2$, then $V^{2}$ decreases toward zero. Moreover, if $V_{0}^{2}>1 / 2$, then $V^{2}$ increases toward unity.

It is important to compare and contrast figures 3 and 4 the curvature is weaker for larger $r_{0}$; therefore, for a given initial $\Gamma_{0}$, it takes longer for the particle's speed to approach the speed of light $(\Gamma \rightarrow \infty)$. In fact, $T_{d}^{2} \propto r_{0}^{3}$ from equation (46), as can be verified based on the data given for figures 3 and 4.

\section{Tidal acceleration}

We have demonstrated in the previous section that tidal acceleration can occur in the tangential directions perpendicular to the $Z$-axis. In our approximation, the non-Newtonian character of the phenomenon of tangential tidal acceleration occurs if the speed of the clump exceeds the critical speed of $1 / \sqrt{2}$. In the three-dimensional case, motion relative to the standard reference trajectory can occur in $X, Y$ and $Z$ directions. The main factors responsible for reversing the Newtonian behavior of the tides along these directions are

$$
\begin{aligned}
& \nu_{X}=1-2 \dot{X}^{2}+\frac{4}{3} \dot{Y}^{2}-\frac{2}{3} \dot{Z}^{2} \\
& \nu_{Y}=1-2 \dot{Y}^{2}+\frac{4}{3} \dot{X}^{2}-\frac{2}{3} \dot{Z}^{2} \\
& \nu_{Z}=1-2 \dot{Z}^{2}+\frac{1}{3}\left(\dot{X}^{2}+\dot{Y}^{2}\right),
\end{aligned}
$$

based on equations (33)- (35). For instance, $\nu_{X}<0$ would imply that the character of the motion along the $X$-direction could be opposite of that expected from the Newtonian gravitation theory.

Observe that $\nu_{X}$ and $\nu_{Y}$ cannot both be negative. This follows from the inequality $\nu_{X}+\nu_{Y}>0$, since

$$
\frac{1}{2}\left(\nu_{X}+\nu_{Y}\right)=\frac{1}{3}\left(1-\dot{X}^{2}-\dot{Y}^{2}\right)+\frac{2}{3}\left(1-\dot{Z}^{2}\right),
$$




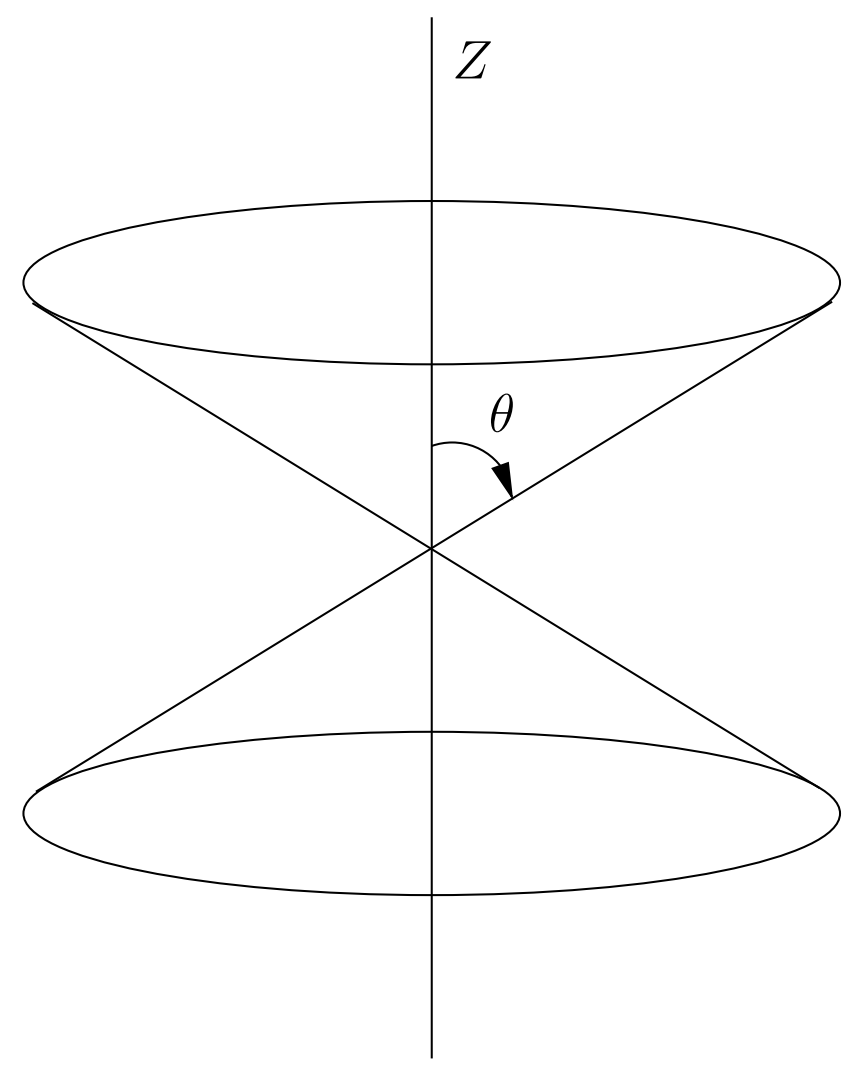

Figure 5: Schematic representation of the boundary cone separating the regions of relative deceleration and acceleration. Particles whose initial velocities are inside the cone decelerate; particles whose velocities are outside the cone accelerate. 
where the right side is a sum of two manifestly positive terms. Moreover, inspection of equations (48)-(50) reveals that

$$
\nu_{X}+\frac{5}{3}>0, \quad \nu_{Y}+\frac{5}{3}>0, \quad \nu_{Z}+1>0 .
$$

On the other hand, it is in general possible to have $\nu_{Z}<0$ and either $\nu_{X}$ or $\nu_{Y}$ also negative. An example of this situation is provided by $\dot{X}=\sqrt{19 / 60}$, $\dot{Y}=0$ and $\dot{Z}=\sqrt{5} / 3$. While the dynamics of this case is complicated, numerical experiments show that $\Gamma$ slowly decreases with time.

It follows from these results that highly relativistic particles emitted near the black hole are accelerated along tangential directions and hence immediately leave the black hole environment, while highly relativistic particles emitted along the rotation axis are decelerated and appear as jets.

We have performed a series of numerical experiments to delineate the regions of acceleration and deceleration of particles relative to the reference particle. The results are represented in figure 5. For $r_{0} /(G M)=100$, numerical experiments show that $\theta$ is independent of $\Gamma_{0}$ and is given approximately by $\tan \theta=\sqrt{2}$, so that $\theta \approx 54.7^{\circ}$. In our numerical experiments, $\theta$ remained approximately constant for $\Gamma_{0} \geq 5$ and $5 \leq r_{0} /(G M) \leq 100$.

\section{Discussion}

The generalized Jacobi equation has been known for three decades [5], but its physical consequences for relativistic relative motion have only recently received attention 1, 2. An important issue regarding the generalized Jacobi equation is that for ultrarelativistic relative motion, the duration of validity of this equation is rather short. However, we have shown that even within this limit, for ultrarelativistic outward motion along the rotation axis of a Kerr black hole, there is a remarkably strong initial tidal deceleration relative to the ambient medium regardless of the value of $\Gamma_{0} \gg 1$. To go beyond this initial deceleration, let us note that a significant feature of our basic equations (8) - (10) is that they do not explicitly depend on the reference trajectory corresponding to a "fixed" marker in the ambient medium. One can therefore imagine a number of such markers along the path of the jet such that from one marker to the next, the integration of equation (12) describes the force-free jet deceleration to a good approximation. In this way, one may approach the terminal speed of the jet by referring the jet motion to different markers. So long as the jet speed relative to any nearby ambient marker is significantly greater than $1 / \sqrt{2}$, it tends to decrease toward this terminal speed. 
It is possible to view the terminal speed in another way. Along a radial direction far from a massive source, its tidal influence on two neighboring test particles is a tidal acceleration if the relative speed of the particles is significantly below $1 / \sqrt{2}$. However, this influence turns to a tidal deceleration if the relative speed is significantly above $1 / \sqrt{2}$.

We have neglected plasma effects in this paper; in fact, our treatment is valid for a force-free plasma. If the plasma is not force-free, the inclusion of its effects would enormously complicate matters [2, 12]. In any case, tidal deceleration is most effective very close to the central source, where the jet originates. Moreover, it is expected to be more significant for microquasars, since tidal effects near a black hole generally decrease as the inverse square of the mass of the source. Thus the results of this paper may well play a significant role in the dynamics of jets, especially in microquasars 4. More generally, this work may be of interest in connection with the astrophysics of energetic particles such as the ultrahigh energy cosmic rays.

Let us now turn to the motion of a particle normal to the rotation axis of the black hole. Relative to the reference particle, the particle accelerates if its initial speed exceeds the critical speed $1 / \sqrt{2}$. For ultrarelativistic relative motion, this tidal acceleration can lead to ultrahigh energy particles with $\Gamma \rightarrow \infty$ according to the generalized Jacobi equation; more generally, particle acceleration occurs outside a cone of angle $\theta$, where the polar angle $\theta$ is measured from the $Z$-axis and $\tan \theta \approx \sqrt{2}$. As the particle accelerates beyond the Fermi system, one can in principle use other overlapping coordinate systems to describe the motion over an extended period of time. On the other hand, the tidal effects of the black hole diminish rapidly with increasing distance and hence the influence of the black hole can be neglected once the particle has moved sufficiently far away.

The rotation of the source leads to a preferred radial direction, i.e. the axis of rotation, but is otherwise of little importance for the tidal dynamics presented in this paper.

The nature of the accretion mechanisms that create ultrarelativistic particles near black holes is beyond the scope of our investigation. The analysis presented in this paper based on the generalized Jacobi equation indicates that once such particles are created, those propagating mainly along the rotation axis of the black hole decelerate with respect to the ambient medium, while those propagating mainly normal to this axis can accelerate to $\Gamma \rightarrow \infty$.

The loss of kinetic energy for the motion of an initially ultrarelativistic particle along the rotation axis and the gain in kinetic energy for motion normal to this axis may be attributed, in analogy with Newtonian gravitation, to the change in the gravitational potential energy, which is the tidal 
energy in the case under consideration in this paper. The underlying assumption here is that the perturbation of the background geometry caused by the motion of the test particle may be neglected. This breaks down, however, when $\Gamma \rightarrow \infty$; therefore, the back-reaction on the motion is expected to moderate this singularity leading to an ultrahigh energy particle. More generally, the gravitational and electromagnetic (in case of charged particles) radiations emitted by the decelerating/accelerating particles should be taken into account as well.

It is well known that primary ultrahigh energy cosmic ray protons with energies above $10^{20} \mathrm{eV}$ from extragalactic sources are expected to interact with the cosmic microwave background photons resulting in photopion production and pair creation [13, 14. Therefore, it may be reasonable to assume that the most energetic particles that reach the solar system originate within our galaxy (see [15] for a recent review of cosmic ray astrophysics). In view of the foregoing results, it would be interesting to determine whether the directional distribution of the ultrahigh energy cosmic rays is correlated with the known microquasars, taking due account of the directionality of the acceleration zones normal to the jet directions.

\section{Appendix A Cylindrical coordinates}

In system (33) - (35), let us introduce the cylindrical coordinates $\rho, \varphi$ and $Z$, where

$$
X=\rho \cos \varphi, \quad Y=\rho \sin \varphi .
$$

The resulting system is given by

$$
\begin{aligned}
\ddot{\rho}- & \rho \dot{\varphi}^{2}-\frac{1}{2} k\left[\rho\left(1-2 \dot{\rho}^{2}+\frac{4}{3} \rho^{2} \dot{\varphi}^{2}-\frac{2}{3} \dot{Z}^{2}\right)+\frac{14}{3} \dot{\rho} Z \dot{Z}\right] \\
& +q \rho \dot{\varphi}(\rho \dot{\rho} \dot{Z}-2 Z)=0 \\
\rho \ddot{\varphi}+ & 2 \dot{\rho} \dot{\varphi}+\frac{1}{3} k \rho \dot{\varphi}(5 \rho \dot{\rho}-7 Z \dot{Z})+q\left[2 \dot{\rho} Z+\rho \dot{Z}\left(1+\rho^{2} \dot{\varphi}^{2}\right)\right]=0 \\
\ddot{Z}+ & k\left\{Z\left[1+\frac{1}{3}\left(\dot{\rho}^{2}+\rho^{2} \dot{\varphi}^{2}\right)-2 \dot{Z}^{2}\right]+\frac{2}{3} \rho \dot{\rho} \dot{Z}\right\} \\
& -q \rho^{2} \dot{\varphi}\left(1-\dot{Z}^{2}\right)=0 .
\end{aligned}
$$

This system depends on $\varphi$ only through $\dot{\varphi}$ and $\ddot{\varphi}$ and is therefore invariant under $\varphi \mapsto \varphi+$ constant, which again reflects the axial symmetry of the system. 
A remarkable feature of system (A2)- (A4) is that for $\dot{\varphi}=0$ and $Z=0$, the system reduces to purely radial motion given by

$$
\ddot{\rho}-\frac{1}{2} k \rho\left(1-2 \dot{\rho}^{2}\right)=0,
$$

which is the same form as equation (12) except for $k \mapsto-k / 2$. This reversal of sign of the curvature leads to the acceleration of an ultrarelativistic particle that is launched from $\rho=0$ in a direction normal to the $Z$-axis as illustrated in figures 3 and 4

It has been shown in [1] that the tidal equation is derived from a Lagrangian given in Fermi coordinates by

$$
L=-\sqrt{-g_{00}-2 g_{0 i} V^{i}-g_{i j} V^{i} V^{j}},
$$

where the metric components in Fermi coordinates are given by equation (6). Using equations (29)-(31), it is possible to show that in our approximation the Lagrangian reduces to

$$
\tilde{L}=-\sqrt{1-\dot{X}^{2}-\dot{Y}^{2}-\dot{Z}^{2}-\frac{1}{2} k(T) S-2 q(T)(X \dot{Y}-\dot{X} Y) Z},
$$

where $S(X, Y, Z, \dot{X}, \dot{Y}, \dot{Z})$ is given by

$$
S=X^{2}+Y^{2}-2 Z^{2}+\frac{2}{3}(X \dot{Y}-\dot{X} Y)^{2}-\frac{1}{3}(X \dot{Z}-\dot{X} Z)^{2}-\frac{1}{3}(Y \dot{Z}-\dot{Y} Z)^{2} .
$$

Expressed in cylindrical coordinates, $\tilde{L}$ takes the form

$$
\tilde{L}=-\sqrt{1-\dot{\rho}^{2}-\rho^{2} \dot{\varphi}^{2}-\dot{Z}^{2}-\frac{1}{2} k(T) \Psi-2 q(T) \rho^{2} \dot{\varphi} Z},
$$

where

$$
\Psi=\rho^{2}-2 Z^{2}+\frac{1}{3} \rho^{2}\left(2 \rho^{2}-Z^{2}\right) \dot{\varphi}^{2}-\frac{1}{3}(\rho \dot{Z}-\dot{\rho} Z)^{2} .
$$

Here $\varphi$ is a cyclic coordinate; therefore,

$$
P_{\varphi}=\frac{\partial \tilde{L}}{\partial \dot{\varphi}}
$$

is an approximate constant of the motion for system (A2)-(A4) given by

$$
P_{\varphi}=\frac{\left[1+\frac{1}{6} k(T)\left(2 \rho^{2}-Z^{2}\right)\right] \rho^{2} \dot{\varphi}+q(T) \rho^{2} Z}{\sqrt{1-\dot{\rho}^{2}-\rho^{2} \dot{\varphi}^{2}-\dot{Z}^{2}-\frac{1}{2} k(T) \Psi-2 q(T) \rho^{2} \dot{\varphi} Z}} .
$$

One can interpret $P_{\varphi}$ as a generalized orbital angular momentum about the $Z$-axis; its approximate constancy in time is related to the axial symmetry of the system (A2)-(A4). We do not know if system (A2)-( 


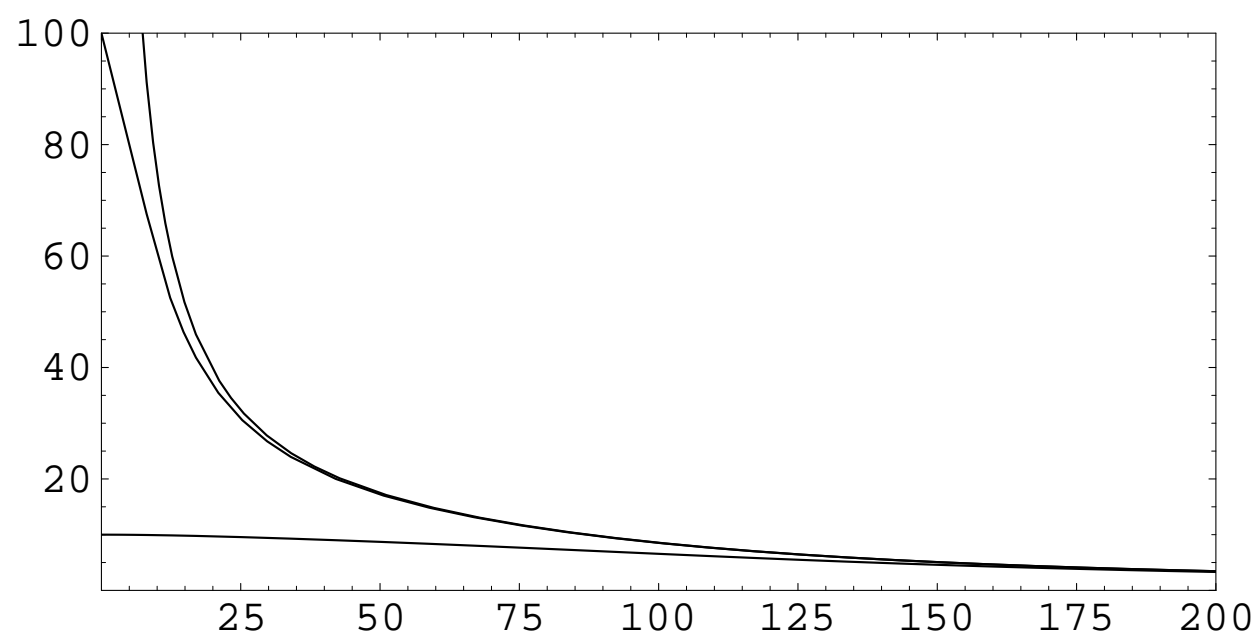

Figure 6: Plot of the Lorentz factor $\Gamma$ versus $T /(G M)$ based on the integration of equation (B1) with initial data $r_{0} /(G M)=100, Z(0)=0$ and $V(0)=\sqrt{\Gamma_{0}^{2}-1} / \Gamma_{0}$ for $a /(G M)=0$. The graph illustrates the deceleration of clumps with initial Lorentz factors $\Gamma_{0}=1000,100$ and 10.

\section{Appendix B Higher-order tidal accelerations}

It is in principle possible to obtain the infinite set of higher-order tidal accelerations that have been ignored in our analysis thus far. The generalized Jacobi equation contains the first-order tidal terms [5]. Tidal terms of second and third order were first calculated in 6] and [7, respectively. Based on these previous detailed calculations, the first two such contributions to equation (9) can be computed and hence the resulting augmented form of equation (9) is

$$
\begin{aligned}
\frac{d^{2} Z}{d T^{2}}= & -\left(k+\frac{1}{2} k^{\prime} Z+\frac{1}{6} k^{\prime \prime} Z^{2}\right)\left(1-2 V^{2}\right) Z+\frac{1}{2}\left(K+\frac{1}{3} K^{\prime} Z\right) V Z^{2} \\
& -\frac{2}{3} k^{2}\left(1+V^{2}\right) Z^{3}+O\left(Z^{4}\right) .
\end{aligned}
$$

Here $V=d Z / d T, k$ is given by equation (10) as before and

$$
\begin{aligned}
k^{\prime}(T) & ={ }^{F} R_{0303 ; 3}=R_{\mu \nu \rho \sigma ; \delta} \lambda_{(0)}^{\mu} \lambda_{(3)}^{\nu} \lambda_{(0)}^{\rho} \lambda_{(3)}^{\sigma} \lambda_{(3)}^{\delta}, \\
k^{\prime \prime}(T) & ={ }^{F} R_{0303 ; 33}=R_{\mu \nu \rho \sigma ; \delta \omega} \lambda_{(0)}^{\mu} \lambda_{(3)}^{\nu} \lambda_{(0)}^{\rho} \lambda_{(3)}^{\sigma} \lambda_{(3)}^{\delta} \lambda_{(3)}^{\omega}, \\
K(T) & ={ }^{F} R_{0303 ; 0}=R_{\mu \nu \rho \sigma ; \delta} \lambda_{(0)}^{\mu} \lambda_{(3)}^{\nu} \lambda_{(0)}^{\rho} \lambda_{(3)}^{\sigma} \lambda_{(0)}^{\delta}, \\
K^{\prime}(T) & ={ }^{F} R_{0303 ; 30}=R_{\mu \nu \rho \sigma ; \delta \omega} \lambda_{(0)}^{\mu} \lambda_{(3)}^{\nu} \lambda_{(0)}^{\rho} \lambda_{(3)}^{\sigma} \lambda_{(3)}^{\delta} \lambda_{(0)}^{\omega},
\end{aligned}
$$




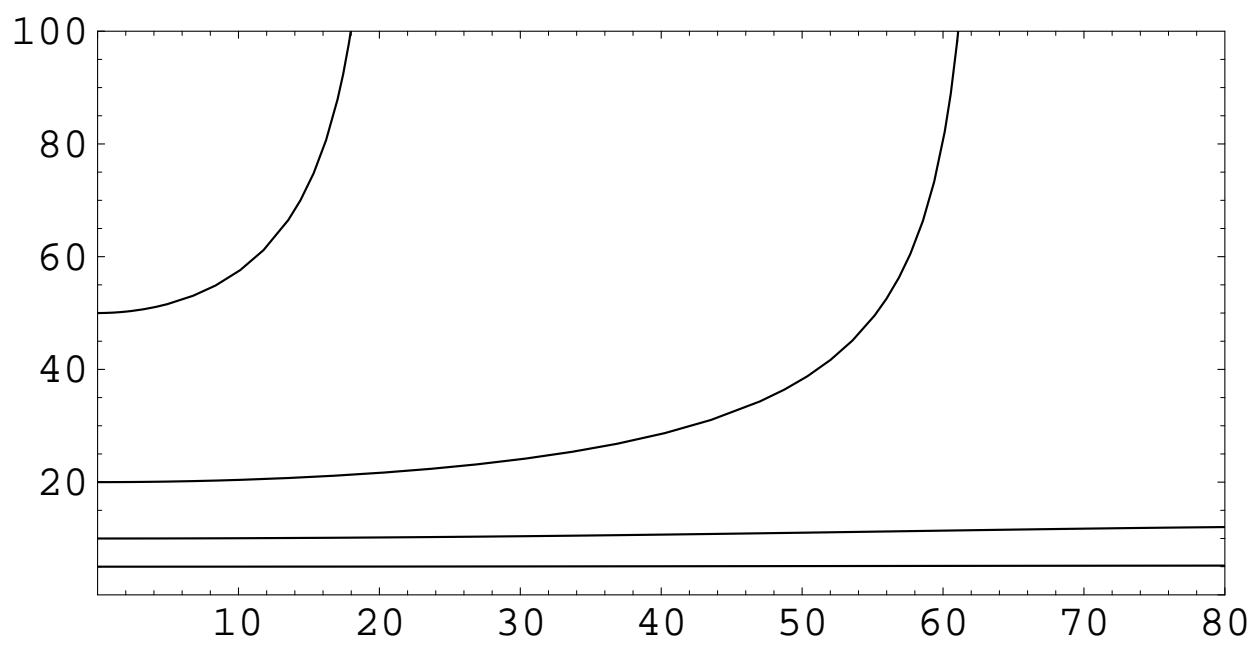

Figure 7: Plot of the Lorentz factor $\Gamma$ versus $T /(G M)$ based on integration of equation (B13) with initial data $X=0$ at $T=0$ with $\dot{X}(0)=2 \sqrt{6} / 5$, $3 \sqrt{11} / 10, \sqrt{399} / 20$ and $7 \sqrt{51} / 50$ corresponding respectively to $\Gamma_{0}=5,10$, 20 and 50. In this plot $a /(G M)=0$ and $r_{0} /(G M)=100$. The graph illustrates acceleration of the particle such that $\Gamma$ essentially approaches infinity at $T /(G M) \approx 63$ and 21 for $\Gamma_{0}=20$ and 50 , respectively. 
along the reference worldline where $\partial x^{\mu} / \partial X^{\alpha}=\lambda_{(\alpha)}^{\mu}$. In principle, the projections of the covariant derivatives of the Riemann curvature tensor of Kerr spacetime on the tetrad frame of the reference particle evaluated along its worldline are required for the determination of the higher-order tidal accelerations. However, for $r_{0} \gg G M$, it is simpler and more instructive to employ the linear approximation of general relativity as follows. The linearized Kerr metric in the post-Newtonian framework is given by

$$
\begin{aligned}
d s^{2}= & -(1+2 \Phi) d t^{2}+\frac{4 G J}{r^{3}}(y d x-x d y) d t \\
& +(1-2 \Phi)\left(d x^{2}+d y^{2}+d z^{2}\right)
\end{aligned}
$$

where $\Phi=-G M / r$ is the Newtonian potential. This metric can be written as $g_{\mu \nu}=\eta_{\mu \nu}+h_{\mu \nu}$, where $h_{00}=-2 \Phi, h_{i j}=-2 \Phi \delta_{i j}$ and

$$
h_{0 i}=-2 G \frac{\mathbf{J} \times \mathbf{r}}{r^{3}}
$$

is proportional to the gravitomagnetic vector potential. The Riemann tensor in the linear approximation is given by

$$
R_{\mu \nu \rho \sigma}=\frac{1}{2}\left(h_{\mu \sigma, \nu \rho}+h_{\nu \rho, \mu \sigma}-h_{\nu \sigma, \mu \rho}-h_{\mu \rho, \nu \sigma}\right),
$$

which can be represented in the standard manner as a symmetric $6 \times 6$ matrix with indices that range over the set $\{01,02,03,23,31,12\}$. For the linearized Kerr metric $(\underline{\mathrm{B} 6})$, we have such a representation in terms of symmetric and traceless $3 \times 3$ matrices $E$ and $B$,

$$
\left[\begin{array}{cc}
E & B \\
B & -E
\end{array}\right]
$$

where $E$ and $B$ are the electric and magnetic parts of the curvature and

$$
\begin{aligned}
E_{i j} & =R_{0 i 0 j}=\Phi_{, i j}=\frac{G M}{r^{3}}\left(\delta_{i j}-3 \frac{x^{i} x^{j}}{r^{2}}\right), \\
B_{i j} & =\frac{1}{2} \epsilon_{j k \ell} R_{0 i k \ell}=-3 \frac{G}{r^{5}}\left[x^{i} J^{j}+x^{j} J^{i}+\left(\delta_{i j}-5 \frac{x^{i} x^{j}}{r^{2}}\right) \mathbf{r} \cdot \mathbf{J}\right] .
\end{aligned}
$$

In the linear approximation under consideration here $\lambda_{(\alpha)}^{\mu}$ along the reference worldline in effect reduces to $\delta^{\mu}{ }_{\alpha}$ in the computation of the Riemann tensor and its covariant derivatives (which reduce to partial derivatives) as measured by the reference particle. It is therefore interesting to 
note that along the reference trajectory $(x=y=0, z=r)$, the Riemann tensor measured by the reference particle turns out to be identical to equations (29)-(31) without the $a^{2}$ terms, as can be verified by direct calculation using (B10) and (B11).

The rotation of the source plays a rather minor role in the numerical results of this paper; therefore, for the sake of simplicity we will set $J=0$ in the computation of $k^{\prime}, k^{\prime \prime}, K$ and $K^{\prime}$. We find that $K=K^{\prime}=0$ and $k=-2 G M / r^{3}$,

$$
k^{\prime}=6 \frac{G M}{r^{4}}, \quad k^{\prime \prime}=-24 \frac{G M}{r^{5}},
$$

so that $k^{\prime}=k_{, 3}$ and $k^{\prime \prime}=k_{, 33}$ along the reference path $(z=r)$. It follows that the results in equation $(\mathbf{B} 12)$ agree with the higher-order Newtonian tidal terms in equation (26). Thus the first term on the right-hand side of (B1) contains the higher-order Newtonian tidal terms once $1-2 V^{2} \mapsto 1$ in the nonrelativistic limit. Equation (BB1) can now be integrated with $r=r(T)$ given by (13); the results of this integration are presented in figure 6] where the Lorentz factor versus $T$ is given for $r_{0} /(G M)=100$ and initial Lorentz factors 1000, 100 and 10. This figure should be compared and contrasted with figure 2

Let us now turn to the higher-order tidal corrections to equation (44). Based on the detailed treatments in [6, 7], the result is

$$
\begin{aligned}
\frac{d^{2} X}{d T^{2}}= & -X\left(1-2 \dot{X}^{2}\right)\left({ }^{F} R_{0101}+\frac{1}{2 !} F_{0101 ; 1} X+\frac{1}{3 !}{ }^{F} R_{0101 ; 11} X^{2}\right) \\
& +\frac{1}{2} X^{2} \dot{X}\left({ }^{F} R_{0101 ; 0}+\frac{1}{3}{ }^{F} R_{0101 ; 10} X\right)-\frac{2}{3} X^{3}\left(1+\dot{X}^{2}\right)\left({ }^{F} R_{0101}\right)^{2} \\
& +O\left(X^{4}\right) .
\end{aligned}
$$

Using our approximation scheme with $J=0$, we find that along the trajectory ${ }^{F} R_{0101 ; 0}={ }^{F} R_{0101 ; 10}=0$ and

$$
{ }^{F} R_{0101}=\frac{G M}{r^{3}}, \quad{ }^{F} R_{0101 ; 1}=0, \quad{ }^{F} R_{0101 ; 11}=-9 \frac{G M}{r^{5}} .
$$

Equation (B13) can be integrated with $r=r(T)$ given by (13); the Lorentz factor versus $T$ is given in figure 7 for $r_{0} /(G M)=100$. The results should be compared and contrasted with those given in figure 4. The terms in equations (B1) and (B13) proportional to $k^{2}$ make negligible contributions to the numerical work presented in figures 6 and 7 respectively, in agreement with our approximation scheme. 
Within the limit of validity of the tidal equation, the higher-order tidal terms provide small corrections to the generalized Jacobi equation as in figures 6]and 7. To demonstrate that the main results of this paper, namely, the strong initial tidal deceleration of ultrarelativistic flows along the rotation axis and the acceleration normal to this axis will remain unchanged by the addition of the higher-order tidal terms, we have integrated equations (B1) and (B13) with the same initial data as in figures 2 and 4 respectively. The results are presented in figures [6] and 7. respectively, which appear to be almost identical to figures 2] and 4, respectively and demonstrate that the higher-order tidal terms slightly enhance the deceleration effect and tend to moderate the acceleration effect. For instance, in figure 2, the graph for initial $\Gamma_{0}=100$ reaches $\Gamma \approx 4.63$ at $T=100 \mathrm{GM}$, while in figure 6] it reaches $\Gamma \approx 3.44$. Nevertheless, figures 6 and 7 demonstrate that the inclusion of higher-order tidal terms will not change the main results of this work.

Concerning the tails in figures 10 2] and 6, we note that so long as the initial $\Gamma_{0}$ is well above the critical value of $\sqrt{2}$, a temporal interval of integration can be found such that the flow decelerates as described in detail in this paper. However, the size of this temporal interval of validity decreases as the flow speed approaches the critical speed. Thus sufficiently close to the critical speed our approach loses its validity. Moreover, based on the available terms of the tidal equation (B1), it is clear that the critical speed $1 / \sqrt{2}$ is not a feature of the general tidal equation in this case. It follows that our treatment breaks down when the jet clump decelerates to a speed sufficiently close to this critical speed. On the astrophysical side, we note that by the time the clump decelerates close to the critical speed relative to the ambient medium, the black hole may be sufficiently far from the clump that tidal effects may no longer play a significant role in the subsequent motion of the clump.

\section{References}

[1] C. Chicone and B. Mashhoon, Class. Quantum Grav. 19, 4231 (2002).

[2] C. Chicone, B. Mashhoon and B. Punsly, Int. J. Mod. Phys. D, in press; astro-ph/0308421 (2003).

[3] J.L. Synge, Relativity: The General Theory, North-Holland, Amsterdam (1960).

[4] R. Fender, Lect. Notes Phys. 589, 101 (2002). 
[5] B. Mashhoon, Astrophys. J. 197, 705 (1975).

[6] B. Mashhoon, Astrophys. J. 216, 591 (1977).

[7] W.Q. Li and W.T. Ni, J. Math. Phys. 20, 1925 (1979).

[8] B. Mashhoon, J.C. McClune and H. Quevedo, Class. Quantum Grav. 16, 1137 (1999).

[9] B. Mashhoon, Phys. Lett. A 163, 7 (1992).

[10] B. Mashhoon and J.C. McClune, Mon. Not. Roy. Astron. Soc. 262, 881 (1993).

[11] J.K. Beem, P.E. Ehrlich and K.L. Easley, Global Lorentzian Geometry, 2nd edn, Dekker, New York, ch 2 (1996).

[12] B. Punsly, Black Hole Gravitohydromagnetics, Springer-Verlag, New York (2001).

[13] K. Greisen, Phys. Rev. Lett. 16, 748 (1966).

[14] G.T. Zatsepin and V.A. Kuzmin, JETP Lett. 4, 78 ( 1966) [Pisma Zh. Eksp. Teor. Fiz. 4, 114 (1966)].

[15] L.A. Anchordoqui, C.D. Dermer and A. Ringwald, hep-ph/0403001 